\title{
A noção de cultura alimentar em ações de educação alimentar e nutricional em escolas brasileiras: uma análise crítica
}

\author{
The notion of food culture in the actions of food and nutrition \\ education in Brazilian schools: a critical analysis
}

Ursula Peres Verthein (https://orcid.org/0000-0002-1304-9642) ${ }^{1}$

Ligia Amparo-Santos (https://orcid.org/0000-0002-6925-6421) ${ }^{2}$

${ }^{1}$ Observatorio de la Alimentación, Departamento de Antropologia Social. Av. Prat de la Riba 171, E-08921, Santa Coloma de Gramenet. Barcelona Espanha. ursulaverthein@gmail.com ${ }^{2}$ Escola de Nutrição, Universidade Federal da Bahia. Salvador BA Brasil.

\begin{abstract}
The scope of this paper is to conduct a critical analysis on how perceptions of the notion of food culture have been presented in scientific papers on food and nutrition education in Brazilian Elementary schools. To achieve this, the use and application of this concept in the texts were recorded and the forms of the proposed relationship between the inclusion of food culture in the theoretical-methodological planning and the practice of the actions were critically read and analyzed. The paper is based on qualitative document research. The theoretical scope of the socio-anthropology of food is used as the focal point to support the reflections. Twenty scientific papers were selected on food and nutrition education published in various databases between 2010 and 2018. For data production and analysis, discourse analysis techniques were used. The conclusion drawn is that, despite being mentioned repeatedly in the texts, food culture is not consolidated as a legitimate dimension in the field of food and nutrition education. Although it is almost always claimed in the texts, it is not always explicitly and concretely mentioned. Therefore, it ends up losing the relevance and weight that, paradoxically, it already has.
\end{abstract}

Key words Food and nutrition education, Culture, School food
Resumo O objetivo deste artigo é analisar criticamente como as compreensões a respeito da noção de cultura alimentar têm sido articuladas em artigos científicos sobre educação alimentar e nutricional (EAN) desenvolvidas em escolas brasileiras da educação básica. Para isso, fez-se um registro dos usos e aplicações desse conceito nos textos e leu-se criticamente as formas de relação propostas entre a inclusão da cultura alimentar no planejamento teórico-metodológico e a prática das ações analisadas. $O$ artigo parte de uma pesquisa qualitativa de base documental. O escopo teórico da socioantropologia da alimentação serve de embasamento para sustentar as reflexões. Para a produção dos dados foram selecionados 20 artigos científicos sobre EAN publicados entre $2010 e$ 2018 em diferentes bases de dados. Para o processo de análise dos dados foram utilizadas técnicas da análise do discurso. Concluiu-se que a cultura alimentar, apesar de mencionada de forma repetida nos textos, não se consolida como uma dimensão legítima no campo da EAN. Ainda que na maior parte das vezes esteja reivindicada nos textos, nem sempre é referenciada de forma explícita e concreta. Por isso, acaba perdendo a relevância e o peso que, paradoxalmente, já tem.

Palavras-chave Educação alimentar e nutricional, Cultura, Alimentação escolar 


\section{Introdução}

O atual consenso sobre as problemáticas decorrentes do crescimento da incidência de sobrepeso e obesidade infantil no mundo, em função dos seus "riscos" e "prejuízos" para a saúde, determina a proposição de ações e estratégias diversificadas para a sua prevenção e cuidado. O consumo alimentar "inadequado" e o "nível insuficiente de atividade física" são as principais causas apontadas para o aumento da ocorrência de doenças crônicas não transmissíveis, em especial a obesidade. É nesse contexto que a educação alimentar e nutricional (EAN) assume relevância fundamental, sendo considerada como instrumento eficaz para a promoção da saúde e a prevenção de doenças, além de ser considerada uma promotora do bem -estar físico e emocional da população $0^{1-8 .}$

No Brasil, Ramos e colaboradores ${ }^{9}$ destacam a relevância assumida pela EAN no âmbito das políticas públicas em alimentação e nutrição, principalmente partir dos anos 1990, quando a "questão da promoção de hábitos alimentares saudáveis passou a constar nos programas oficiais brasileiros, a exemplo da Política Nacional de Alimentação e Nutrição (PNAN)"10. A partir de então, dentro do contexto nacional de promoção da EAN, destacam-se diversas iniciativas como o Programa Saúde nas Escolas, por meio do Decreto $\mathrm{n}^{\circ} 6.286 / 2007^{11}$, que tem como objetivo promover ações relacionadas a prevenção, atenção e promoção da saúde dos estudantes, incluindo a alimentação saudável. Além disso, há o Marco de Referência de Educação Alimentar e Nutricional para as Políticas Públicas (2012) ${ }^{12}$, o atual Guia alimentar para a população brasileira ${ }^{13}$, as ações de EAN inseridas no Programa de Nacional de Alimentação Escolar (PNAE) ${ }^{14} \mathrm{e}$ ou a Lei $n^{\circ} 16.333 / 2018^{15}$, que inclui a educação alimentar e nutricional nas escolas como tema transversal.

O Marco de Referência de Educação Alimentar e Nutricional para as Políticas Públicas ${ }^{12}$ define que a EAN, “[...] no contexto da realização do Direito Humano à Alimentação Adequada e da garantia da Segurança Alimentar e Nutricional, é um campo de conhecimento e de prática contínua e permanente, transdisciplinar, intersetorial e multiprofissional que visa promover a prática autônoma e voluntária de 'hábitos alimentares saudáveis"” (p. 23).

O Guia alimentar para a população brasileira $^{13}$, por sua vez, define o propósito de estabelecer diretrizes para apoiar as ações em EAN e alerta: "as estratégias de educação alimentar e nutricional devem apoiar pessoas, famílias e comunidades para que adotem práticas alimentares promotoras da saúde [...]" (p. 22). Em relação ao $\mathrm{PNAE}^{14}$, as ações de EAN se referem, por exemplo, à promoção de oferta de alimentação adequada $\mathrm{e}$ saudável na escola; ao incentivo à formação de pessoas envolvidas direta ou indiretamente com a alimentação escolar; à dinamização do currículo das escolas, tendo por eixo temático a alimentação e a nutrição; ao incentivo e promoção de hábitos alimentares regionais e culturais saudáveis ou à utilização do alimento como ferramenta pedagógica.

Evidencia-se, portanto, que a EAN assume papel fundamental e crescente na prevenção e no cuidado do sobrepeso e da obesidade na população infantil. O campo científico (em diversas áreas como a saúde, a educação e as ciências sociais e humanas) e o setor público, no planejamento e execução de políticas públicas, reivindicam e promovem de forma crescente a centralidade da EAN como meio de difusão e consolidação dos conhecimentos sobre alimentação "adequada" e "saudável" que desejam difundir.

No entanto, estudos analíticos sobre as ações de EAN desenvolvidas na atualidade diagnosticaram distâncias entre a teoria e a prática e incoerências entre o planejamento e a execução das atividades. E como causas desses discrepâncias, identificaram problemáticas como: inconsistência das bases teórico-metodológicas que servem de apoio ao desenvolvimento das atividades; grande utilização de métodos expositivos, prescritivos e normatizadores que desconsideram a perspectiva dialógica de base freiriana da educação (que paradoxalmente costumam reivindicar); inadequação de técnicas e materiais pedagógicos utilizados; formação insuficiente dos profissionais envolvidos na execução das ações; distância das propostas em relação às realidades e especificidades locais dos grupos; descontinuidade das atividades; temporalidade insuficiente; dificuldade de diálogo entre atores envolvidos; inoperância, na prática, da intersetorialidade prevista nos projetos e planejamentos ${ }^{16-19}$

No que se refere especificamente à trajetória de aproximação da cultura à alimentação escolar no contexto brasileiro, podemos destacar três momentos relevantes. Primeiro, o movimento de descentralização que ocorre a partir do início do processo de redemocratização do país. Esse processo possibilita uma relativa autonomia das atividades e ações no que diz respeito à contextualização e à "regionalização" da abordagem teórico-metodológica e da práxis da educação. Além disso, a 
inclusão da noção de cultura na Lei Nacional de Segurança Alimentar e Nutricional (Lei no 11.346, de 15 de setembro de 2006$)^{20}$, em sua definição, já no artigo $2, \$ 1^{\circ}$, sobre a necessidade de que as políticas e ações de segurança alimentar e nutricional sejam construídas e postas em prática levando-se "em conta as dimensões ambientais, culturais, econômicas, regionais e sociais". Além disso, um terceiro elemento relevante nessa aproximação cultura-alimentação escolar refere-se à Lei $\mathrm{n}^{\circ}$ 11.947, de 16 de junho de $2009^{14}$, que determina que pelo menos $30 \%$ do valor repassado pelo governo Federal a estados, municípios e Distrito Federal pelo Fundo Nacional de Desenvolvimento da Educação (FNDE) para o Programa Nacional de Alimentação Escolar (PNAE) deve ser para compra de alimentos oriundos "da agricultura familiar e do empreendedor familiar rural ou de suas organizações, priorizando-se os assentamentos da reforma agrária, as comunidades tradicionais indígenas e as comunidades quilombolas"14.

Dado o processo de aproximação que vai se construindo no cenário brasileiro entre a alimentação escolar e a noção de cultura (e cultura alimentar, consequentemente), em função dos fatores acima referenciados, nos cabe questionar quais as compreensões sobre o conceito têm sido utilizadas nesse contexto. Assim, este artigo tem como objetivo analisar criticamente como as publicações referenciam a dimensão da cultura alimentar. Essas reflexões colaboram com as análises a respeito das formas de aplicação da noção de cultura alimentar na construção teórico-metodológica e na prática das ações de EAN nas escolas de educação básica.

\section{Metodologia}

Esse artigo parte de uma pesquisa qualitativa de base documental. O escopo teórico da socioantropologia da alimentação ${ }^{21-24}$ serve de embasamento para sustentar as reflexões.

Quanto ao levantamento bibliográfico de artigos científicos (revisados por pares) sobre educação alimentar e nutricional em escolas brasileiras, este concentrou-se nas publicações realizadas entre os anos 2010 e 2018, pela sua atualidade. A busca foi feita nas seguintes bases de dados: Lilacs e Medline, considerando o enfoque da saúde; Scielo, para trazer à discussão leituras realizadas por outras áreas sobre o tema (como educação ou ciências sociais e humanas); e nos periódicos da CAPES e na Biblioteca Virtual em Saúde (BVS), a fim de identificar artigos não en- contrados na primeira etapa do levantamento bibliográfico. Para a busca bibliográfica, foram utilizados os seguintes descritores: "educação alimentar e nutricional" e "escola”. Depois de uma primeira etapa de busca abrangente, foi realizada a análise de títulos e resumos, com o objetivo de verificar se os artigos se encaixavam nos critérios de inclusão. Por fim, foram selecionados 20 artigos científicos que correspondiam aos critérios estipulados $^{25-44}$ (Quadro 1).

Além dos critérios de inclusão já referidos, a pertinência dos artigos selecionados para a construção dessa argumentação se justifica porque os mesmos abordam assuntos relevantes para pensar o planejamento e a prática das ações sobre EAN. Por exemplo: (a) importância da ludicidade para o processo de ensino e aprendizagem em $\mathrm{EAN}^{33,35,40}$; (b) utilização de oficinas culinárias como recurso pedagógico ${ }^{32}$; (c) verificação da "efetividade" de intervenções nutricionais em escolares na "modificação do consumo alimentar" 34,36,39, no "conhecimento sobre alimentação e nutrição" 36 e no "estímulo do consumo de alimentos" $^{43}$; (d) a interprofissionalidade ${ }^{31,38,41,42}$; (e) a transdiciplinaridade ${ }^{37}$; (f) a formação de profissionais em alimentação escolar ${ }^{28,29}$; $\mathrm{g}$ ) ações educativas sobre alimentação orgânica ${ }^{30}$; ou (h) o uso de hortas escolares como prática educativa ${ }^{26,27}$.

Para preservar os autores, optou-se neste artigo por não referenciar explicitamente a origem dos fragmentos transcritos ao citarmos os textos analisados (exemplo: páginas 6, 7 e 8). No entanto, para garantir sua legitimidade, sabe-se que esses mesmos trechos provêm do corpus supracitado $^{25-44}$.

Para cumprir os objetivos propostos, fez-se um registro dos usos e aplicações da noção de cultura alimentar nos artigos científicos selecionados. Além disso, leu-se criticamente a relação entre essas formas de inclusão e a teoria e prática das ações analisadas. Para o processo de análise, foi realizada uma aproximação interpretativa própria da análise do discurso, por sua capacidade de leitura da informação para além do texto em si. Nessa abordagem, o processo hermenêutico e a construção dos sentidos se dão a partir da inserção dos dados em relação com seus contextos (social, econômico, cultural etc. $)^{45,46}$. A análise dos dados foi realizada por meio de leitura e interpretação dos textos a partir do objetivo proposto pela pesquisa. Para isso, mapeou-se no corpus do arquivo (20 artigos científicos) as formas como a cultura alimentar foi referenciada (em suas referências diretas e a partir de conceitos derivados). Ao mesmo tempo, estabeleceu-se 
Quadro 1. Artigos selecionados para análise.

\begin{tabular}{|c|c|c|}
\hline Autoria & Título & $\begin{array}{c}\text { Revista/ano de } \\
\text { publicação }\end{array}$ \\
\hline $\begin{array}{l}\text { Anzolin C, Ouriques CM, } \\
\text { Höfelmann DA, Mezadri T }\end{array}$ & Intervenções nutricionais em escolares & RBPS/2010 \\
\hline Coelho DEP, Bógus CM & $\begin{array}{l}\text { Vivências de plantar e comer: a horta escolar como } \\
\text { prática educativa, sob a perspectiva dos educadores }\end{array}$ & Saúde Soc/2016 \\
\hline $\begin{array}{l}\text { Costa MC, Sampaio EV, Zanirati } \\
\text { VF, Lopes AC, Santos LC }\end{array}$ & $\begin{array}{l}\text { Experiência lúdica de promoção de alimentação } \\
\text { saudável no ambiente escolar: satisfação e aprendizado } \\
\text { dos estudantes }\end{array}$ & $\begin{array}{l}\text { Mundo da } \\
\text { saúde/2016 }\end{array}$ \\
\hline $\begin{array}{l}\text { Fernandes AGS, Fonseca ABC, da } \\
\text { Silva, AA. }\end{array}$ & $\begin{array}{l}\text { Alimentação escolar como espaço para a educação em } \\
\text { saúde: percepção das merendeiras do município do Rio } \\
\text { de Janeiro, Brasil }\end{array}$ & $\begin{array}{l}\text { Cien Saude } \\
\text { Colet/2014 }\end{array}$ \\
\hline $\begin{array}{l}\text { Friedrich RR, Schuch I, Wagner } \\
\text { MB }\end{array}$ & $\begin{array}{l}\text { Efeito de intervenções sobre o índice de massa corporal } \\
\text { em escolares }\end{array}$ & $\begin{array}{l}\text { Rev. Saúde } \\
\text { Pública/2012 } \\
\end{array}$ \\
\hline Gomes, KS, Fonseca ABC & $\begin{array}{l}\text { Dialogando sobre as possibilidades e desafios das } \\
\text { merendeiras nas ações de educação alimentar e } \\
\text { nutricional }\end{array}$ & Demetra/2018 \\
\hline Juzwiack, CR & $\begin{array}{l}\text { Era uma vez... Um olhar sobre o uso dos contos de fada } \\
\text { como ferramenta de educação alimentar e nutricional }\end{array}$ & $\begin{array}{l}\text { Interf Comun } \\
\text { Saude Educ2013 }\end{array}$ \\
\hline $\begin{array}{l}\text { Juswiak CR, de Castro PM, } \\
\text { Batista SH }\end{array}$ & $\begin{array}{l}\text { A experiência da Oficina Permanente de Educação } \\
\text { Alimentar e em Saúde (OPEAS): formação de } \\
\text { profissionais para a promoção da alimentação saudável } \\
\text { nas escolas }\end{array}$ & $\begin{array}{l}\text { Cien Saude } \\
\text { Colet/2013 }\end{array}$ \\
\hline Kops NL, Zys J, Ramos M & $\begin{array}{l}\text { Educação alimentar e nutricional da teoria à prática: } \\
\text { um relato de experiência }\end{array}$ & $\begin{array}{l}\text { Cienc \& } \\
\text { Saúde/2013 } \\
\end{array}$ \\
\hline $\begin{array}{l}\text { Oliveira SRML, Villar BS, Florido } \\
\text { JMP, Schwartzman F, Bicalho D. }\end{array}$ & $\begin{array}{l}\text { Implantação de hortas pedagógicas em escolas } \\
\text { municipais de São Paulo }\end{array}$ & Demetra/2018 \\
\hline $\begin{array}{l}\text { Pereira TS, Pereira, RC, Angelis- } \\
\text { Pereira MC }\end{array}$ & $\begin{array}{l}\text { Influência de intervenções educativas no conhecimento } \\
\text { sobre alimentação e nutrição de adolescentes de uma } \\
\text { escola pública }\end{array}$ & $\begin{array}{l}\text { Cien Saude } \\
\text { Colet/2017 }\end{array}$ \\
\hline $\begin{array}{l}\text { Pires PF, Retondario A, Almeida } \\
\text { CC, Schmidt ST, Beux MR, } \\
\text { Ferreira SMR }\end{array}$ & $\begin{array}{l}\text { Professional practice of dietitians in the Brazilian } \\
\text { School Feeding Program: a multiple case study }\end{array}$ & Rev. Nutri/2017 \\
\hline $\begin{array}{l}\text { Rangel CN, Nunn R, Dysarz F, } \\
\text { Silva E, Fonseca AB }\end{array}$ & $\begin{array}{l}\text { Ensinando e aprendendo sobre alimentação e nutrição } \\
\text { através da educação em ciências: uma interseção de } \\
\text { conhecimentos }\end{array}$ & $\begin{array}{l}\text { Cienc Saude } \\
\text { Colet/2014 }\end{array}$ \\
\hline Rezende MF, Negri ST & $\begin{array}{l}\text { Educação alimentar e nutricional associada a oficinas } \\
\text { culinárias com alunos em uma escola pública }\end{array}$ & $\begin{array}{l}\text { R. Eletr. de } \\
\text { Extensão/2015 }\end{array}$ \\
\hline $\begin{array}{l}\text { Santos LAS, Carvalho DMM, } \\
\text { Reis ABC, Ramos LB, Freitas } \\
\text { MCS }\end{array}$ & $\begin{array}{l}\text { Formação de coordenadores pedagógicos em } \\
\text { alimentação escolar: um relato de experiência }\end{array}$ & $\begin{array}{l}\text { Cien Saude } \\
\text { Colet } / 2013\end{array}$ \\
\hline $\begin{array}{l}\text { Santos VF, Araújo IA, Pires CR, } \\
\text { Kato HC, Sousa DN }\end{array}$ & $\begin{array}{l}\text { Educação alimentar e nutricional para o estímulo } \\
\text { do consumo de pescado por escolares: relato de } \\
\text { experiencia }\end{array}$ & $\begin{array}{l}\text { Rev Elo Diál } \\
\text { Ext / } 2018\end{array}$ \\
\hline Santos VF, Pires CR & $\begin{array}{l}\text { Ludicidade em educação alimentar e nutricional no } \\
\text { âmbito escolar: uma alternativa de prática pedagógica }\end{array}$ & R. Interd/2018 \\
\hline $\begin{array}{l}\text { Silva SU, Monego ET, Sousa LM, } \\
\text { Almeida GM }\end{array}$ & $\begin{array}{l}\text { As ações de educação alimentar e nutricional e o } \\
\text { nutricionista no âmbito do Programa Nacional de } \\
\text { Alimentação Escolar }\end{array}$ & $\begin{array}{l}\text { Cien Saude } \\
\text { Colet/2018 }\end{array}$ \\
\hline $\begin{array}{l}\text { Souza RH, Tomasi CD, Birollo } \\
\text { IV, Ceretta LB, Ribeiro RS }\end{array}$ & Educação alimentar e nutricional: relato de experiência & $\begin{array}{l}\text { Rev Progr } \\
\text { Res Multiprof } \\
\text { Saúde Colet } \\
\text { UNESC/2016 } \\
\end{array}$ \\
\hline $\begin{array}{l}\text { Vieira TV, Corso AC, González- } \\
\text { Chica, DA }\end{array}$ & $\begin{array}{l}\text { Ações educativas sobre alimentação orgânica } \\
\text { desenvolvidas por nutricionistas em escolas municipais } \\
\text { brasileiras }\end{array}$ & Rev Nutr/2014 \\
\hline
\end{tabular}

Fonte: Elaborado pelos autores. 
relações comparativas dos sentidos de cultura alimentar para identificar, entre os textos analisados, aproximações e diferenças nesse sentido.

Portanto, foi a partir desse contexto que foram analisados como os textos científicos estão utilizando a noção de cultura alimentar e as ideias que dela se originam ("identidades", "tradição", "comportamentos alimentares", "hábitos alimentares", "sistemas alimentares" etc.). Além disso, questionou-se como essa aproximação entre comida e cultura é referenciada na construção das práticas das ações educativas.

Em relação às questões éticas, cabe considerar que o projeto de pesquisa que dá origem à produção desse artigo foi devidamente submetido e aprovado pelo Comitê de Ética em Pesquisa da Escola de Nutrição da Universidade Federal da Bahia (UFBA).

\section{Resultados e discussão}

\section{O conceito de cultura alimentar}

Para realizar uma reflexão crítica sobre a forma como o conceito de cultura alimentar vem sendo usado como referencial teórico para o planejamento e as práticas das ações de EAN, é preciso retomá-lo. Parte-se da definição assumida por Contreras e Gracia ${ }^{21}$ porque entende-se que ela traz a dimensão e a abrangência necessárias para a abordagem proposta neste artigo, no sentido que defendemos quanto à sua compreensão a partir da complexidade do ato alimentar por meio de suas dimensões: biológica, social e simbólica $^{21-24}$. Assim, segundo Contreras e Gracia ${ }^{21}$, cultura alimentar é: "Conjunto de representaciones, de creencias, conocimientos y prácticas heredadas y o aprendidas que están associadas a la alimentación y que son compartidas por los indivíduos de una cultura dada o de un grupo social determinado dentro de una cultura. Al compartir una cultura, tendemos a actuar de forma similar, a gobernarnos por orientaciones, preferencias y sanciones autorizadas por esta" (p. 37).

Essa definição traz questões importantes para serem pensadas e incluídas em uma análise crítica das atividades de educação relacionadas à comida e ao comer. Por exemplo: a reflexão sobre a necessidade das práticas alimentares serem contextualizadas (no tempo, no espaço, nas relações de poder, nas condições econômicas, sociais e históricas, entre outros aspectos); a colocação de que os determinantes dos comportamentos alimentares surgem dessa contextualização; a consideração acerca do compartilhamento de "crenças", "conhecimentos" e "práticas" associadas à alimentação, que nos leva a pensar criticamente a respeito da construção de nossas identidades individuais e coletivas; a discussão sobre quais saberes, técnicas e sabores legitimamos e quais estigmatizamos; a inserção das prescrições e proibições alimentares em um contexto determinado.

Nesse sentido, é preciso considerar que as ideias tratadas no fragmento anterior sobre o conceito de cultura alimentar se enquadram na própria definição do conceito de EAN, segundo o Marco de Referência de Educação Alimentar e Nutricional para as políticas públicas ${ }^{12}$, e não se dissociam da perspectiva assumida por ele: "A adoção de um conceito de EAN deve considerar aspectos que contemplem desde a evolução histórica e política da EAN no Brasil às múltiplas dimensões da alimentação e do alimento e os diferentes campos de saberes e práticas conformando uma ação que integre o conhecimento científico ao popular. Adota-se o termo Educação Alimentar e Nutricional e não o termo Educação Nutricional ou o termo Educação Alimentar para que o escopo de ações abranja desde os aspectos relacionados ao alimento e alimentação, os processos de produção, abastecimento e transformação aos aspectos nutricionais" (p. 23).

Um primeiro dado a ser discutido é o fato de que, constatou-se, as ações de EAN costumam por vezes centrar sua abordagem sobre a alimentação em sua dimensão biológica. Além disso, nessa perspectiva, evidencia-se um caráter medicalizador, e portanto normativo ou prescritivo, do ato alimentar ${ }^{47}$. Em função desse aspecto, as ações tendem a deixar em segundo plano a dimensão social da alimentação. Assim, apesar da cultura ser um elemento valorizado pelas políticas públicas e pelos trabalhos científicos em EAN, o planejamento e a prática das ações podem ainda não incorporar de forma complexa os conhecimentos desse campo.

Os objetivos das atividades analisadas muitas vezes relatam processos de aprendizagem centrados na transmissão de conhecimentos que visam difundir padrões alimentares e estilos de vida "saudáveis" ou "adequados". Nesse sentido, a ideia de "alimentação saudável" está fundamentalmente centrada no "nutriente". Por vezes, ainda que as narrativas sobre essas atividades façam referência a práticas que "contribuem no conhecimento dos alimentos" ou que "estimulam a autonomia de escolares para escolhas alimentares saudáveis", revela-se com frequência uma perspectiva intervencionista sobre o corpo e o 
comportamento do outro, expresso no sentido de "construir", "controlar", "transformar" "hábitos alimentares" e "padrões de consumo alimentar" dos alunos. Nessa linha, em muitos casos, o objetivo central expresso pelas abordagens de EAN assumidas nos textos que analisamos é a "saúde", o "emagrecimento" ou a "adoção de práticas alimentares saudáveis".

No mesmo sentido, os resultados relatados por esses artigos se concentram muitas vezes nos mesmos aspectos. Os textos apontam, por exemplo, que as atividades foram capazes de gerar "conhecimentos adequados sobre nutrição"; que as "intervenções nutricionais foram efetivas na modificação do consumo de alguns alimentos/grupos alimentares", ou que colaboram na obtenção de "modificações efetivas no consumo alimentar".

\section{A noção de cultura alimentar na escola segundo as publicações do campo de EAN}

No contexto problematizador das ações de EAN, nos centramos então em um aspecto concreto para reflexão. Apesar da referência comum à dimensão biológica do comer no desenvolvimento das propostas de EAN analisadas, por sua relação imediata habitual com a promoção e o cuidado à saúde na escola, a cultura alimentar é um elemento com grande relevância na teoria sobre as ações educativas nessa área na atualidade. Percebe-se que os textos científicos que abordam questões diversas relativas a planejamentos e atividades de EAN utilizam a noção de cultura alimentar repetidamente, seja para tratar da sua importância como elemento de "construção" do repertório alimentar de indivíduos ou grupos, para reivindicar a "preservação" das identidades regionais por meio da alimentação, para incluir a alimentação na transversalidade disciplinar requerida pelas políticas públicas de saúde e educação, para promover a contextualização e adequação das propostas às realidades locais, ou para promover recomendações de cardápios adequados às especificidades de cada lugar, entre outras formas de referência.

Em relação a esse aspecto, destaca-se, por exemplo, que o Marco de Referência de Educação Alimentar e Nutricional para Políticas Públicas ${ }^{12}$ se refere a questões diversas. Trata da importância da cultura como determinante das escolhas alimentares individuais e coletivas; da relação comida-identidade; entende que as ações serão mais efetivas se abordarem a estrutura social de forma abrangente, trazendo para a discussão a interferência dos contextos de produção, distribuição e consumo da nossa comida; aborda a "Valorização da cultura alimentar local e respeito à diversidade de opiniões e perspectivas, considerando a legitimidade dos saberes de diferentes" (p. 25), entre outras formas de trazer e propor a relevância do campo para a construção das "estratégias" educativas.

Um exemplo concreto de como a noção de cultura se expressa nos textos analisados ${ }^{25-44}$ elucida as questões tratadas até aqui. Uma das experiências relatadas constrói essa aproximação alimento-cultura. As autoras iniciam a introdução de seu trabalho abordando os comportamentos e as tradições alimentares como campos dinâmicos e contextualizados historicamente. Além disso, tratam das transformações nos modelos alimentares e dos consequentes impactos nas identidades e nas relações sociais nos meios rurais e urbanos. Citando autores da socioantropologia da alimentação, como Jesús Contreras, Mabel Gracia, Claude Fischler e Jean-Pierre Poulain, abordam ainda fenômenos da contemporaneidade, como a ruptura de vínculo entre o alimento, a natureza e o consequente processo que denominam de padronização dos alimentos. Além disso, abordam questões relativas à perda de identidade ou qualidade simbólica do alimento nesse contexto. O texto menciona outras questões do campo sociantropológico da alimentação, como a redução da importância social da alimentação em situações determinadas e os processos de flexibilização de normas que regem o comer e a diversidade de discursos sobre a alimentação. O texto comenta também a importância da escola como espaço privilegiado para síntese entre a cultura própria dos alunos e a cultura formal, institucionalizada, destacando a importância do papel da escola nesse sentido.

Nesse contexto, percebe-se como a cultura alimentar é referenciada e convive com perspectivas teórico-metodológicas diversas de EAN, mesmo as que se apresentam como contra-hegemônicas, como aquelas que se alinham às ações de caráter normativo-prescritivo para a saúde e o corpo. No entanto, apesar de a cultura assumir nos textos sobre EAN sentidos tão diversos, por vezes ela é utilizada de forma pouco complexa, a ponto de sua relevância não ser justificada ou até mesmo de ser contradita.

Em um dos artigos selecionados, na introdução os autores utilizam concepções diferentes em um mesmo parágrafo, de tal forma que assumem uma postura divergente. Iniciam afirmando que a "educação nutricional" exige o de- 
senvolvimento de abordagens pedagógicas que possibilitem a interpretação do comer a partir de sua complexidade, englobando assim a multidimensionalidade de alimentação. No entanto, nesse mesmo parágrafo, defendem ser função do nutricionista ajudar as pessoas a modificarem seus hábitos e práticas alimentares, por meio da assistência nutricional individual ou coletiva. Assim, posicionam-se primeiro a favor de uma expansão do campo para os contextos, mas acabam por reduzir a questão à responsabilidade individual e à "mudança de hábitos". Por isso, os "problemas alimentares" (apesar de não estarem claramente definidos no texto, pressupõe-se sua equivalência às práticas alimentares "não saudáveis") passam de uma questão ampla e relacionada à cultura a uma questão individual e de responsabilidade de um único profissional (nutricionista). A respeito desse aspecto da responsabilidade profissional sobre a EAN, cabe considerar que as políticas, a exemplo do Marco de Referência de Educação Alimentar e Nutricional para Políticas Públicas ${ }^{12}$ colocam-se a favor da intersetorialidade e da interdisciplinaridade.

Em outro relato acerca de "intervenções nutricionais” realizadas em alunos, na introdução do texto os autores iniciam um parágrafo afirmando que a "promoção da saúde" na escola exige uma abordagem integral e multidisciplinar, o que implica, segundo os mesmos, considerar contextos ampliados, como a família, a comunidade, a sociedade e o ambiente onde estão inseridos os sujeitos. Apesar dessa referência aos contextos, o relato sobre as atividades de intervenção se concentra basicamente na transmissão de conhecimento a respeito da "alimentação saudável”. As atividades propostas são ações de caráter fundamentalmente informativo sobre "pirâmide alimentar" ou valor nutritivo de alimentos para "escolhas saudáveis". Além disso, o método de avaliação empregado para medir a "efetividade" se concentra, segundo relatado no texto, na comparação de frequências e número de vezes que as crianças consumiram os diferentes alimentos, antes e depois das ações. É importante perceber que, nesse caso concreto, apesar de o contexto macrossocial ser considerado um fator determinante das escolhas e do consumo alimentar, tanto a abordagem teórico-metodológica das atividades como o método de avalição da "efetividade" das mesmas são definidas sem a inclusão apropriada da cultura alimentar.

A leitura dos textos científicos no campo demonstra, portanto, que os mesmos utilizam o conceito de cultura alimentar repetidamente, abordando-o de forma difusa. A maneira como é abordado o conceito nos textos acaba por definir uma concepção abrangente, mas por vezes não de modo claro ou concreto. Outra questão importante a considerar é que os textos nem sempre se referem explicitamente ao conceito de "cultura alimentar" para abordá-lo. Ela é citada em suas dimensões e tratada como "hábitos alimentares", "comportamentos alimentares", "fatores sociais", "hábitos alimentares regionais", "práticas alimentares (saudáveis)", "preferências alimentares", "atitudes exibidas pelos adultos" ou “a língua deles[alunos]”, por exemplo. Em muitas ocasiões, ao incluírem esses conceitos, os textos não os relacionam diretamente à teoria da cultura e, por conseguinte, não os fundamentam de forma complexa. Assim, a cultura alimentar, apesar de ser mencionada repetidamente nos textos, não se consolida como uma dimensão legítima no campo da EAN. Na verdade, ela quase sempre está reivindicada nos textos, mas nem sempre é referenciada de modo explícito e concreto. Por isso, acaba perdendo a relevância e o peso que, tácita e paradoxalmente, já tem.

Ao mesmo tempo, o conceito, abordado de forma diversa, algumas vezes define com pouca precisão sua aplicação na prática cotidiana das ações educativas. Percebe-se que, por exemplo, o termo é utilizado: como referência às particularidades locais na alimentação de grupos sociais; para abordar questões diversas relativas às identidades e "tradições" regionais; para incluir a alimentação na escola de forma transversal e interdisciplinar; para fomentar a adequação das propostas educativas aos contextos (regionais, econômicos, sociais, históricos etc.) dos alunos; e para estimular a implementação de cardápios adequados às especificidades de cada lugar entre outras. No entanto, nem sempre essa diversidade se concretiza na prática das atividades.

\section{Considerações finais}

Vê-se que há nessa aproximação dos textos sobre a EAN e a cultura alimentar uma utilização difusa do conceito. Ele, não sendo mencionado em todas as ocasiões como tal, é tratado também a partir de ideias, expressões e conceitos diversos (como "hábitos", “comportamentos", “identidades", “aspectos regionais" etc). Seu uso repetido e extenso nem sempre corresponde com uma ampla aplicação na prática das ações educativas. Além disso, pode ocorrer de a cultura alimentar ser mencionada como base para a construção teórico-me- 
todológica das propostas educativas nos textos científicos que as relatam, mas ser paradoxalmente desconsiderada por esses mesmos textos. Esse status da cultura alimentar na forma como vem sendo considerada na educação revela, portanto, uma tentativa de inseri-la nas discussões, a fim de torná-las mais complexas e aprofundadas. No entanto, a maneira como tem sido considerada na sua relação teoria-práxis apresenta os desafios que ainda temos nesse caminho.

Conclui-se que, apesar da referência à cultura alimentar aparecer (de forma direta ou indireta) nos artigos analisados, não costuma ocorrer um aprofundamento sobre as questões nesse sentido. Além disso, não há necessariamente relação entre o uso repetido do conceito de cultura alimentar nos trabalhos e a sua real utilização na prática das atividades. As ações concretas nem sempre se beneficiam do repertório que a cultura alimentar, reivindicada na teoria, poderia conceder às práticas das ações de EAN.

Nesse caminho da aproximação entre a alimentação escolar e a cultura, a escola pode abrir espaços para questões diversas, como soberania e segurança alimentar, direito humano à alimentação adequada, desigualdade social, formas de produção, distribuição e consumo alimentar, estigmatização do corpo, obesidade, prazer de comer, comensalidade, sustentabilidade e agroecologia. Essas questões podem fazer parte das propostas educativas porque, além dos conteúdos que propõem reflexões fundamentais, tratam das condições sociais em que são forjados os "hábitos" e "comportamentos alimentares" que estão sendo questionados e categorizados como "incorretos".

A partir dessa perspectiva, a cultura alimentar é necessariamente parte da educação alimentar e nutricional, pois traz para o centro das ações as relações sociais concretas que permeiam o ato alimentar. Por isso, se considerarmos a materialidade dos contextos nas nossas discussões (e práticas) sobre o comer, sua inserção no campo da EAN viabiliza a proposta reivindicada pelos diversos atores implicados nos planejamentos e nas práticas das ações.

\section{Colaboradores}

Ambas as autoras trabalharam na concepção, metodologia, pesquisa, análise de dados, redação e revisão final.

\section{Agradecimento}

Ao Conselho Nacional de Desenvolvimento Científico e Tecnológico (CNPq), pela bolsa de Pós-doutorado Junior concedida, que permitiu, no desenvolvimento do projeto de pesquisa, a produção deste artigo. 


\section{Referências}

1. Allirot X, Quinta N, Chokupermal K, Udaneta E. Involving children in cooking activities: a potential strategy for directing food choices toward novel food containing vegetables. Appetite 2016; 103:275-285.

2. Birch L, Savage JS, Ventura A. Influences on the development of children's eating behaviors: from infancy to adolescence. Can J Diet Pract Res 2007; 68(1):s1s56.

3. Mello ED, Luft VC, Meyer F. Obesidade infantil: como podemos ser eficazes. J Pediatr (Rio J) 2004; 80(3):173-182.

4. Oliveira AMA, Cerqueira EMM, Oliveira AC. Prevalência de sobrepeso e obesidade infantil na cidade de Feira de Santana-BA: detecção na família $\mathrm{x}$ diagnóstico clínico. J Pediatr (Rio J) 2003; 79(4):325-328.

5. Oliveira CL, Fisberg M. Obesidade na infância e adolescência - uma verdadeira epidemia. Arq Bras Endocrinol Metab 2003; 47(2):107-108.

6. Pérez EC, Sandoval MJ, Schneider SE, Azula LA. Epidemiología del sobrepeso y la obesidad en niños y adolescentes. Rev Post Via Cat Med 2008; 179:16-20.

7. Gallardo LP, Bayona I, Mingo, T, Rubiales C. Utilidad de los programas de educación nutricional para prevenir la obesidad infantil a través de um estudio piloto em Soria. REV Nutr Hosp 2011; 26(5):1161-1167.

8. Vio F, Salinas J, Montenegro E, González CG, Lera I. Efecto de una intervención educativa em alimentación saludable em profesores y niños preescolares y escolares de la región de Valparaíso, Chile. Nutr Hosp 2014; 29(6):1298-1304.

9. Ramos F, Reis A, Santos L. Educação alimentar e nutricional em escolares: uma revisão de literatura. Cad Saude Publica 2013; 29(11):2147-2161.

10. Brasil. Ministério da Saúde (MS). Secretaria de Atenção à Saúde. Departamento de Atenção Básica. Política Nacional de Alimentação e Nutrição. Brasília: MS; 2013.

11. Brasil. Decreto no 6.286, de 5 de dezembro de 2007. Institui o Programa Saúde na Escola- PSE e dá outras providências. Diário Oficial da União 2007; 6 dez.

12. Brasil. Secretaria Nacional de Segurança Alimentar e Nutricional. Ministério do Desenvolvimento Social e Combate à Fome. Marco de referência de educação alimentar e nutricional para as políticas públicas. Brasília: Ministério do Desenvolvimento Social e Combate à Fome; 2012.

13. Brasil. Ministério da Saúde (MS). Secretaria de Atenção à Saúde. Departamento de Atenção Básica. Guia alimentar para a população brasileira. Brasília: MS; 2014.

14. Brasil. Lei no 11.947 , de 16 de junho de 2009. Dispõe sobre o atendimento da alimentação escolar e do Programa Dinheiro Direto na Escola aos alunos da educação básica. Altera as Leis no 10.880 , de 9 de junho de 2004, 11.273, de 6 de fevereiro de 2006, 11.507, de 20 de julho de 2007; revoga dispositivos da Medida Provisória no 2.178-36, de 24 de agosto de 2001, e a Lei $n^{\circ} 8.913$, de 12 de julho de 1994; e dá outras providências. Diário Oficial da União 2009; 17 jun.
15. Brasil. Lei $\mathrm{n}^{\circ} 16.333$, de 16 de maio de 2018. Altera a Lei no 9.394, de 20 de dezembro de 1996 (Lei de Diretrizes e Bases da Educação Nacional), para incluir o tema transversal da educação alimentar e nutricional no currículo escolar. Diário Oficial da União 2018; 17 maio.

16. Santos LA. O fazer educação alimentar e nutricional: algumas contribuições para reflexão. Cien Saude Colet 2012; 17(2):453-462.

17. Boog M. Educação nutricional: passado, presente, futuro. R Nutr Puc Camp 1997; 10(1):5-19.

18. Aguiar O, Barão G, Padrão S. Educação alimentar e nutricional: a defesa de uma perspectiva contra-hegemônica e histórico-crítica para educação. Demetra 2017; 12(3):665-682.

19. Yepes T. Desde la educación para la salud: hacia la pedagogía de la educación alimentaria y nutricional. Perspec Nutr Hum 2016; 16:21-40.

20. Brasil. Lei Nacional de Segurança Alimentar e Nutricional. Lei no 11.346 , de 15 de setembro de 2006. Cria o Sistema Nacional de Segurança Alimentar e Nutricional (SISAN) com vistas a assegurar o direito humano à alimentação adequada e dá outras providências. Diário Oficial da União 2006;

21. Contreras J, Gracia M. Alimentación y cultura: perspectivas antropológicas. Barcelona: Ariel; 2005.

22. Fischler C. El (h)omnívoro: el gusto, la cocina, el cuerpo. Barcelona: Anagrama; 1995.

23. Montanari M. La comida como cultura. Gijón: Trea; 2006.

24. Poulain J, organizador. Dictionnaire des cultures alimentaires. Paris: PUF; 2012.

25. Kops NL, Zys J, Ramos M. Educação alimentar e nutricional da teoria à prática: um relato de experiência. Rev Cienc Saude 2013; 6(2):135-140.

26. Coelho DEP, Bógus CM. Vivências de plantar e comer: a horta escolar como prática educativa, sob a perspectiva dos educadores. Saude Soc 2016; 25(3):761-771.

27. Oliveira SRML, Villar BS, Florido JMP, Schwartzman F, Bicalho D. Implantação de hortas pedagógicas em escolas municipais de São Paulo. Demetra 2018; 13(03):583-603.

28. Juswiak CR, Castro PM, Batista SH. A experiência da Oficina Permanente de Educação Alimentar e em Saúde (OPEAS): formação de profissionais para a promoção da alimentação saudável nas escolas. Cien Saude Colet 2013; 18(4):1009-1018.

29. Santos LAS, Carvalho DMM, Reis ABC, Ramos LB, Freitas MC. Formação de coordenadores pedagógicos em alimentação escolar: um relato de experiência. Cien Saude Colet 2013; 18(4):993-1000.

30. Vieira TV, Corso AC, González-Chica DA. Ações educativas sobre alimentação orgânica desenvolvidas por nutricionistas em escolas municipais brasileiras. Rev Nutr 2014; 27(5):525-535.

31. Fernandes AGS, Fonseca ABC, Silva AA. Alimentação escolar como espaço para a educação em saúde: percepção das merendeiras do município do Rio de Janeiro, Brasil. Cien Saude Colet 2014; 19(1):39-48.

32. Rezende MF, Negri ST. Educação alimentar e nutricional associada a oficinas culinárias com alunos em uma escola pública. R Eletr de Extensão 2015; 12(20):21-35. 
33. Costa MC, Sampaio EV, Zanirati VF, Lopes AC, Santos LC. Experiência lúdica de promoção de alimentação saudável no ambiente escolar: satisfação e aprendizado dos estudantes. Mundo da Saúde 2016; 40(1):3850 .

34. Anzolin C, Ouriques CM, Höfelmann DA, Mezadri T. Intervenções nutricionais em escolares. RBPS 2010; 23(4):297-306.

35. Juzwiack CR. Era uma vez... Um olhar sobre os usos dos contos de fada como ferramenta de educação alimentar e nutricional. Interf Comun Saude Educ 2013; 17(45):473-484.

36. Pereira TS, Pereira, RC, Angelis-Pereira MC. Influência de intervenções educativas no conhecimento sobre alimentação e nutrição de adolescentes de uma escola pública. Cien Saude Colet 2017; 22(2):427-435.

37. Rangel CN, Nunn R, Dysarz F, Silva E, Fonseca AB. Ensinando e aprendendo sobre alimentação e nutrição através da educação em ciências: uma interseção de conhecimentos. Cienc Saude Colet 2014; 19(9): 3915-3924.

38. Silva SU, Monego ET, Sousa LM, Almeida GM. As ações de educação alimentar e nutricional e o nutricionista no âmbito do Programa Nacional de Alimentação Escolar. Cienc Saude Colet 2018; 23(8):26712681.

39. Friedrich RR, Schuch I, Wagner MB. Efeito de intervenções sobre o índice de massa corporal em escolares. Rev Saude Publica 2012; 46(3):551-560.

40. Santos VF, Pires CR. Ludicidade em educação alimentar e nutricional no âmbito escolar: uma alternativa de prática pedagógica. $R$ Interd 2018; 11(2):63-69.

41. Gomes KS, Fonseca AB. Dialogando sobre as possibilidades e desafios das merendeiras nas ações de educação alimentar e nutricional. Demetra 2018; 13(1):5568.
42. Pires PF, Retondario A, Almeida CC, Schmidt ST, Beux MR, Ferreira SM. Professional practice of dietitians in the Brazilian School Feeding Program: a multiple case study. Rev Nutri 2017; 30(4):449-510.

43. Santos VF, Araújo IA, Pires CR, Kato HC, Sousa DN. Educação alimentar e nutricional para o estímulo do consumo de pescado por escolares: relato de experiência. Rev Elo Dial Ext 2018; 7(1):46-53.

44. Souza RH, Tomasi CD, Birollo IV, Ceretta LB, Ribeiro RS. Educação alimentar e nutricional: relato de experiência. Rev Progr Res Multiprof Saude Colet 2016; 3.

45. Martínez MM. Ciencia y arte em la metodologia cualitativa. México: Trillas; 2002.

46. Careganato RC, Mutti R. Pesquisa qualitativa: análise de discurso versus análise de Conteúdo. Texto Cont Enferm 2006; 15(4):679-684.

47. Gracia M. Comer bien, comer mal: la medicalización del comportamento alimentario. Sal Publ Mex 2007; 49(3):236-242.

Artigo apresentado em 07/10/2019

Aprovado em 20/04/2020

Versão final apresentada em 22/04/2020

Editores-chefes: Romeu Gomes, Antônio Augusto Moura da Silva 